\title{
Glia Modulate the Response of Murine Cortical Neurons to Excitotoxicity: Glia Exacerbate AMPA Neurotoxicity
}

\author{
L. L. Dugan, ${ }^{1,2, a}$ V. M. G. Bruno, ${ }^{1, b}$ S. M. Amagasu, ${ }^{1}$ and R. G. Giffard ${ }^{1}$ \\ Departments of ${ }^{1}$ Anesthesia and ${ }^{2}$ Medicine, Division of Endocrinology, Gerontology, and Metabolism, Stanford \\ University School of Medicine, Stanford, California 94305
}

We have developed "pure" neuronal cultures $(<1 \%$ astrocytes) from mouse neocortex to study the effect of glial cells on the response of neurons to injury. Cortical neurons were found to require glial-conditioned medium to survive. Immature neurons, 2-4 d in vitro, deprived of glialconditioned medium, underwent apoptosis over $48 \mathrm{hr}$, as suggested by condensed nuclear morphology, DNA fragmentation, and protection by inhibition of macromolecular synthesis. Apoptosis Induced by trophic factor deprivation has been described for other neuronal populations, such as superior cervical ganglion and dorsal root ganglion cells. Cortical neurons in pure culture provide another neuronal population for the study of apoptosis induced by trophic factor deprivation. We then studied the interaction of neurons and glia under excitotoxic conditions. Experiments on mature cultures showed that pure neuronal cultures were at least 10-fold more sensitive to acute glutamate exposure than were neuronal-glial ("mixed") cocultures. The difference in sensitivity between pure neurons and mixed cultures was reduced when mixed cultures were treated with the glutamate uptake inhibitor, L-trans-pyrrolidine-2,4-dicarboxylic acid (trans-PDC). In $24 \mathrm{hr}$ exposure to $\mathrm{N}$-methyl-D-aspartate (NMDA), or oxygen, glucose deprivation, pure neurons were more sensitive than mixed cultures; trans-PDC again increased the sensitivity of mixed cultures to nearly that of pure neuronal cultures. In contrast, mixed and pure neuronal cultures exposed to NMDA for $10 \mathrm{~min}$, or to kainate for $24 \mathrm{hr}$, had similar injury doseresponse curves, suggesting that glial glutamate uptake is a less important protective mechanism in these excitotoxic injuries. Surprisingly, pure neurons were less sensitive than mixed cultures to (RS)-amino-3-hydroxy-5-methyl-4Isoxazolepropionic acid (AMPA) toxicity at concentrations up to $100 \mu \mathrm{m}$. This does not reflect astrocyte toxicity, as AMPA at concentrations to $1 \mathrm{~mm}$ did not injure astrocyte

\footnotetext{
Received July 8. 1994; revised Jan. 19, 1995; accepted Feb. 7, 1995

L.L.D. and V.M.G.B. contributed equally to this report. We thank Xiaoyun Sun for superb tcchnical assistance, Dr. Mark Goldberg for early discussions on pure neuronal cultures, and Dorothy Turetsky for help with histochemistry and photomicrography. This work was supported by a Dana Research Fellow ship and an AGRI grant from the Hartford Foundation to L.L.D., and NIH Grants NSO1425 and GM49831 to R.G.G.

Correspondence should be addressed to Rona G. Giffard, Ph.D. M.D., Department of Anesthesia Research Labs, Room S-272. Stanford University Medical Center, Stanford, CA 94305-5123.

Present address: Department of Neurology, Washington University School of Medicine. St. Louis, MO 63110

Present address: Institute of Pharmacology, University of Catania, Catania, Italy.

Copyright 1995 Society for Neuroscience $0270-6474 / 95 / 154545-11 \$ 05.00 / 0$
}

cultures. Glial cultures showed increased levels of glutamate in the extracellular medium in response to exposure to AMPA, but not NMDA or kainate. However, pure neuronal and mixed cultures exposed to the same concentration of AMPA did not have elevated levels of glutamate in the media. We found that glia were generally neuroprotective under excitotoxic conditions, likely through their ability to clear extracellular glutamate. However, the presence of glia exacerbated AMPA neurotoxicity.

[Key words: astrocytes, glutamate, ischemia, NMDA, kainate, cell culture, neocortex, AMPA]

Glutamate receptor-mediated excitotoxicity is believed to contribute to neuronal injury in many disease states, including CNS trauma and hemorrhage, neurodegenerative diseases, and stroke (Choi and Rothman, 1990; Meldrum and Garthwaite, 1990). Non-neuronal cells, in particular, astrocytes, may modify neuronal responses to excitotoxicity (Rosenberg and Aizenman, 1989; Sugiyama et al., 1989; Hewett et al., 1994) and anoxia (Vibulsreth et al., 1987). Glia may provide substrates for neuronal energy metabolism (Quach et al., 1978; Fox et al., 1988; Swanson et al., 1990), and are important in removing glutamate from the extracellular space. Rosenberg and Aizenman (1989) have shown that a reduction of the number of glia in neuronal glial cocultures (from $90 \%$ to $30 \%$ ) increases the sensitivity of neurons to glutamate toxicity 100 -fold, and glia protect neurons from death due to glutamine in culture medium by uptake of nonenzymatically generated glutamate (Rosenberg, 1991). Neurons, in turn, may modify the homeostatic function of glia. Volterra et al. (1992), reported decreased reuptake of glutamate by astrocytes in response to arachidonic acid, which can be released from neurons (Sanfeliu et al., 1990). Interactions between glia and neurons may be critical in determining the outcome of injury. To address questions about glial-neuronal interactions during injury, studies of neurons and glia separately complement studies carried out in mixed cultures.

Although there are several published methods for growing cortical neuronal cell cultures from mouse or rat brain (Yu et al., 1984; Borg et al., 1985; Rosenberg and Aizenman, 1989), most of these pure cultures still contained a significant percentage of astrocytes $(10-60 \%)$, or were maintained for only short durations, though highly purified neuronal cultures from rat brain have been reported (Aizenman and deVellis, 1987). Instead of focusing on using a chemically defined medium, we used glial-conditioned medium to support the culture of essentially pure mouse cortical neurons to complement the relatively pure astrocyte and mixed neuronal-glial cultures already in use in our laboratory. Glial-conditioned medium was essential for survival 


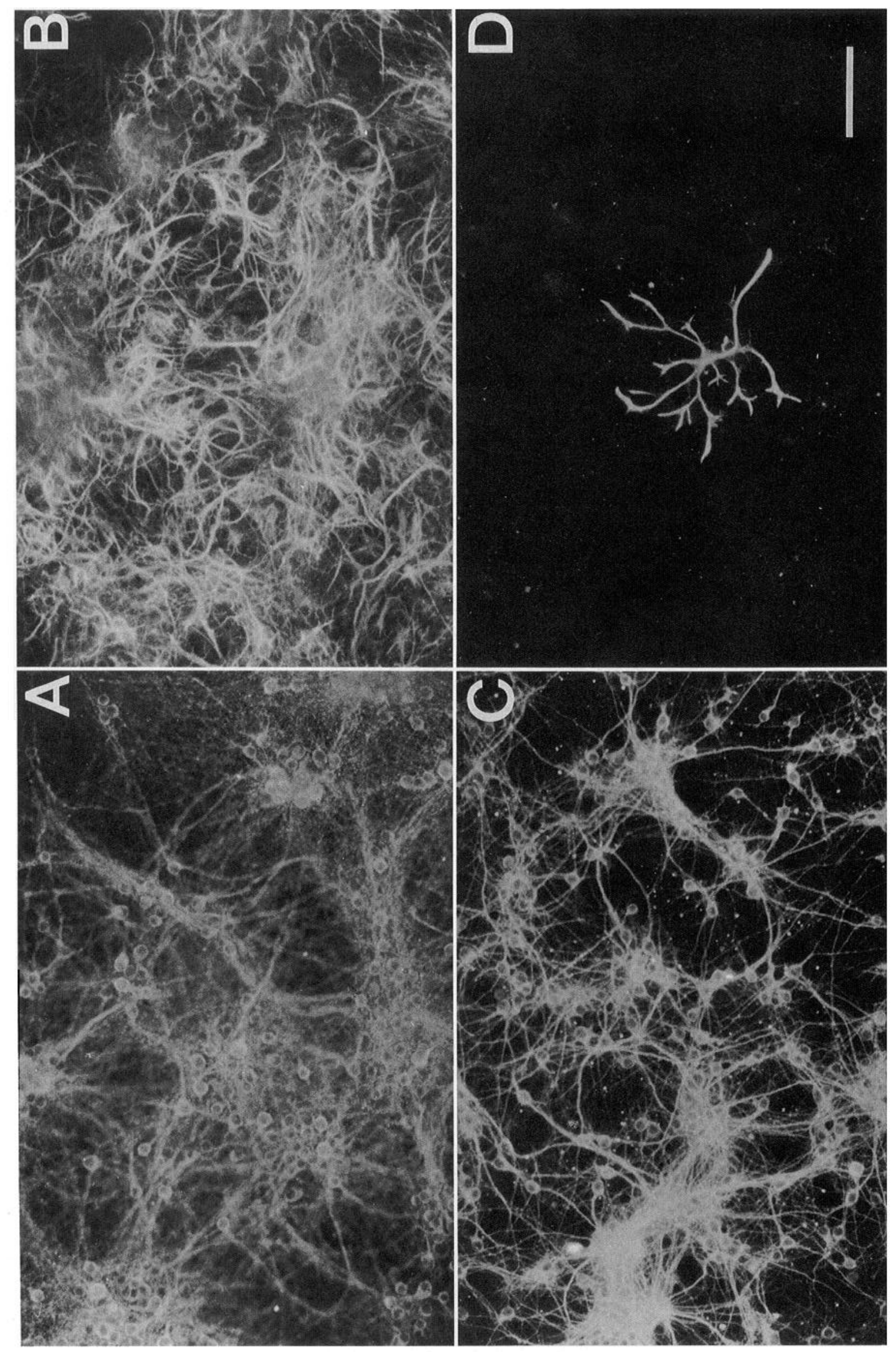

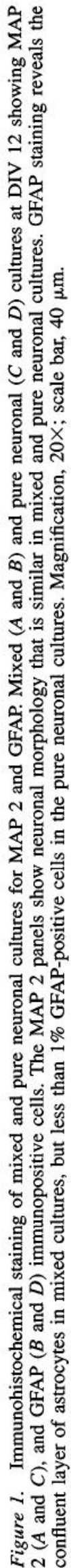


Table 1. Cell counts of neurons and glia in mixed and pure cortical neuronal cultures

\begin{tabular}{llllr} 
& \multicolumn{2}{l}{ NSE-positive cells } & & \multicolumn{2}{c}{ GFAP-positive cells } \\
\cline { 2 - 3 } \cline { 5 - 6 } & DIV 2 & DIV 12 & DIV 2 & DIV 12 \\
\hline Mixed cultures & 3450 & 4250 & 10,050 & 9870 \\
Neuronal cultures & 3020 & 2460 & 22 & 11
\end{tabular}

Mixed cortical cultures and pure neuronal cultures were stained for neuronspecific enolase (NSE) and glial fibrillary acidic protein (GFAP) at DIV 2 and DIV 12. Cells counted in five microscope fields are shown for each condition.

of immature cultures. These cultures were characterized histologically and developmentally, and their response to injury was assessed.

An abstract has appeared (Giffard et al., 1992).

\section{Materials and Methods}

Cell cultures. Pure neuronal cell cultures were prepared from fetal mice at 14-15 d gestation (Dichter, 19/8; Choi et al., 1987) with the following modifications. The brains were removed from fetal mice, freed of meninges. and the cortical hemispheres removed from the rest of the brain. Neocortices were minced and treated with $0.09 \%$ trypsin for 20 $\min$ at $37^{\circ} \mathrm{C}$. After centrifugation at $4000 \mathrm{rpm}$ for $3 \mathrm{~min}$, the pellet was resuspended in $2 \mathrm{ml} \mathrm{PM}$ by gentle trituration through a flame-polished pasteur pipette. The cell suspension was diluted with PM and plated at $400 \mu \mathrm{l}$ per well, 3.5 hemispheres (approximately $2 \times 10^{7}$ cells) per 24 well culture plate; these polystyrene culture plates had been previously coated with poly-D-lysine $(100 \mathrm{ng} / \mathrm{ml})$ and laminin $(4 \mathrm{ng} / \mathrm{ml}$, in water) overnight, washed with water, then MEM, and stored at $4^{\circ} \mathrm{C}$ until use. Twenty-four to $48 \mathrm{hr}$ after plating, half of the culture medium was replaced with GCM, and cytosine arabinoside (Ara-C, $3 \mu \mathrm{M}$ ) was added to each well. Cultures were kept at $37^{\circ} \mathrm{C}$ in a humidified incubator with $5 \% \mathrm{CO}_{2}$ atmosphere without further medium changes. Except as noted, cells were used on days in vitro (DIV) 11-15.

Mixed cortical cultures were prepared in identical fashion through the trituration step. The cell suspension was then plated at 3.5 hemispheres per 24-well plate on plates containing a confluent monolayer of astrocytes. Astrocyte cultures were prepared by plating cortical cell suspensions from 1-2-d-old mouse pups, I cortical hemisphere/24 multiwell Primaria plate (Rose et al., 1993). The mixed cortical cultures were fed twice weekly with MEMglc with $10 \%$ horse serum (first three feedings), and then MEMglc. The astrocyte cultures were fed once per week, and were generally confluent within 2 weeks. Mixed cultures were used for experinients at DIV 13-15.

Immunohistochemistry. For photomicrography, cultures were stained for microtubule-associated protein 2 (MAP 2), and glial fibrillary acidic protein (GFAP). Cells were washed twice with PBS (phosphate-buffered saline), fixed for $30 \mathrm{~min}$ with $4 \%$ paraformaldehyde, washed three times with PBS, then permeabilized with $0.25 \%$ Triton X-100 in PBS for 10 min. Cells were washed, blocked with serum, then incubated with the primary antibody for $24 \mathrm{hr}$ at $4^{\circ} \mathrm{C}$, at $1: 300$ for anti-MAP 2, or 1:10 for anti-GFAP. After washing three times, secondary antibody, labeled with either BODIPY or $\mathrm{Cy} 3$, was added for $30 \mathrm{~min}$ at room temperature in the dark. After three final washes the cells were photographed using a Nikon Diaphot microscope equipped with a mercury lamp.

For cell counts, cultures were stained for neuron-specific enolase (NSE 1:100) and glial fibrillary acidic protein (GFAP 1:10) at DIV 2 and DIV 12. Cells were washed, then incubated in biotinylated antimouse IgG at 1:100 dilution for $30 \mathrm{~min}$. After reaction with avidin and biotin-horseradish peroxidase ( $\mathrm{ABC}$ kit, Vector Labs, Burlingame, CA) staining was developed by exposure to $0.05 \%$ diaminobenzidine $/ 0.01 \%$ $\mathrm{H}_{2} \mathrm{O}_{2}(2-10 \mathrm{~min})$.

Staining for NADPH-diaphorase was performed as described by Koh and Choi (1988), based on the original protocol of Scherer-Singler et al. (1983). Microglia were identified using the lectin, Banderiera simplicifolia I, coupled to horseradish peroxidase. After 30 min incubation at room temperature with lectin at 1:50 dilution, lectin binding was identified using $0.05 \% \mathrm{DAB} / 0.01 \% \mathrm{H}_{2} \mathrm{O}_{2}$ (Colton et al., 1992). Cobalt staining for calcium-conducting AMPA/kainate receptors was done as previously described (Pruss et al., 1991).

DNA fragmentation and nuclear morphology. DNA fragmentation

\section{Table 2. Development of sensitivity to $10 \mathrm{~min}$ NMDA exposure}

\begin{tabular}{cc} 
Age in culture (d) & $\%$ Maximum LDH release \\
\hline 8 & $14.7 \pm 2.8$ \\
11 & $86.6 \pm 8.9^{*}$ \\
14 & $100 \pm 18.8^{*}$
\end{tabular}

Values shown are mean $\pm \operatorname{SEM}(n=4)$. LDH release from pure neuronal cultures of the indicated ages, $24 \mathrm{hr}$ after $10 \mathrm{~min}$ exposure to $300 \mu \mathrm{m}$ NMDA. expressed as percentage of LDH released by freeze-thaw of the same cultures. * Different from day 8 value by ANOVA and Student-Newman-Keuls test. / $<0.05$.

was assessed by $3^{\prime}$ end labeling as described previously (Tilly and Hsueh, 1993). In brief, 24 or 48 hr after being washed free of GCM, the overlying medium was removed while rapidly freezing the cell layer on dry ice. DNA was extracted, $3^{\prime}$ end labeled (500 ng DNA per reaction) with $\alpha^{32}$ P-dideoxy-ATP using terminal dcoxynucleotidyl transferase. The labeled DNA was separated on $2 \%$ agarose gels and visualized by autoradiography using Kodak X-OMAT AR film (Rochester, NY).

Nuclear morphology was assessed at the level of light microscopy on live cultures using the nuclear stain Hoechst dye 33258 (Wallen et al., 1983; Belizario et al., 1993) at $5 \mu \mathrm{g} / \mathrm{ml}$ final concentration added for $10-15 \mathrm{~min}$ at $37^{\circ} \mathrm{C}$ to allow uptake before observation with a Nikon Diaphot microscope equipped for epifluorescence with UV filterblock. Brightly fluorescent staining nuclei either showing a single highly condensed mass of chromatin or lobulated condensed chromatin were scored as apoptotic, compared to dimmer, diffusely fluorescent normal nuclei.

Deprivation of glial-conditioned medium. Pure neuronal cultures on DIV 2 or 3 were washed free of GCM by four medium exchanges using MEMglc. Control cultures were washed an equal number of times with glial-conditioned medium, and additional control sister cultures were observed without any medium exchange. Cultures were assayed for LDH release into the medium to quantitate injury (Koh and Choi, 1987) using a UVmax platereader (Molecular Devices, Menlo Park, CA), stained with Hoechst dye or solubilized for analysis of DNA fragmentation, 24 and $48 \mathrm{hr}$ later. Cultures deprived of glial-conditioned medium in the presence of actinomycin D, $0.3 \mu \mathrm{M}$, or cycloheximide, $0.3 \mu \mathrm{M}$, had the drugs added in the last medium exchange.

Brief exposure to NMDA or glatamate. The culture medium was exchanged twice with HRSS, then glutamate or NMDA was added in the final wash. In experiments with the glutamate uptake inhibitor, trumsPDC (Bridges et al., 1991), $100 \mu \mathrm{M}$ drug was added at the beginning of the $10 \mathrm{~min}$ exposure to glutamate or NMDA, and was present both during the exposure and during the subsequent $24 \mathrm{hr}$. Agonist exposure was performed at room temperature in room air for 10 min. After 10 min, the agonists were washed out by triple exchange of the medium with HBSS (with $5.5 \mathrm{~mm}$ glucose), followed by double exchange with MEMglc. The cultures were kept at $37^{\circ} \mathrm{C}$ in a $5 \% \mathrm{CO}_{2}$ incubator: injury was assessed after 24 hr by microscopic examination, and quantified by measuring lactate dehydrogenase (LDH) efflux. LDH efflux has been shown to be proportional to the number of dead cells assessed by Trypan blue staining (Koh and Choi, 1987). The amount of LDH released by sham-washed controls was subtracted to give the LDH signal specific to the insult, and $24 \mathrm{hr} \mathrm{I} . \mathrm{DH}$ values are expressed as a fraction of the

\begin{tabular}{|c|c|}
\hline \multicolumn{2}{|c|}{$\begin{array}{l}\text { Table } 3 .{ }^{45} \mathrm{Ca}^{2} \text { Accumulation during } 10 \mathrm{~min} \text { exposure to } \mathrm{NMD} \text {. } \\
\text { or glutamate }\end{array}$} \\
\hline Condition & ${ }^{45} \mathrm{Ca}^{2+}$ accumulation (cpm) \\
\hline Control & $84 \pm 13$ \\
\hline NMDA $0.5 \mathrm{~mm}$ & $545 \pm 44^{*}$ \\
\hline Glutamate $0.5 \mathrm{~mm}$ & $373 \pm 45 *$ \\
\hline Kainate $1 \mathrm{~mm}$ & $121 \pm 5$ \\
\hline
\end{tabular}

Values shown are mean $\pm \operatorname{SEM}(n=4)$.

* Significant difference from control by ANOVA and Student-Newman-Keuls test, $p<0.05$. 


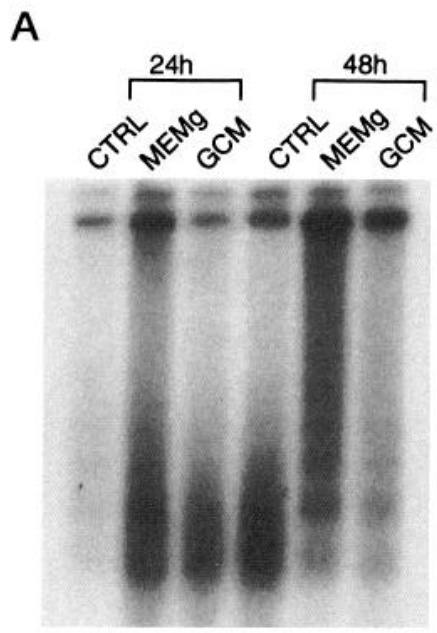

B
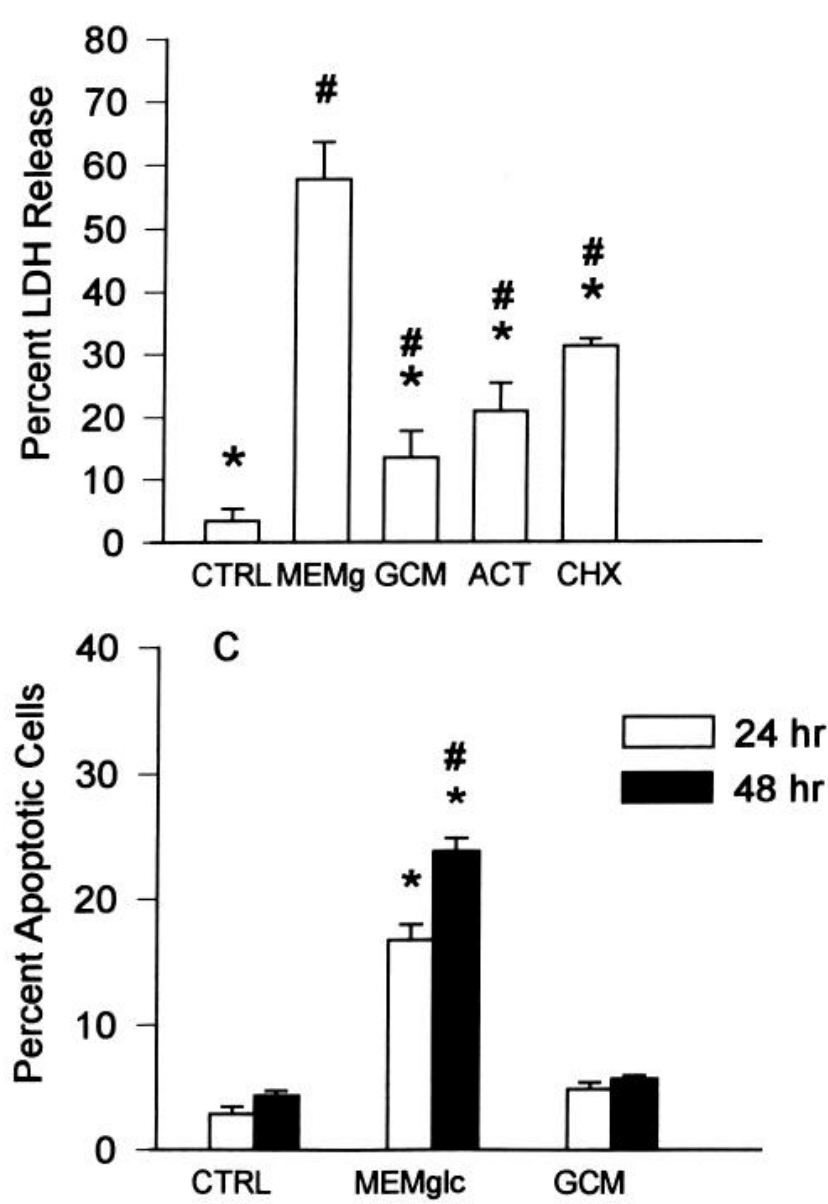

Figure 2. Effects of deprivation of glial-conditioned medium on young neuronal cultures. A, Autoradiogram showing DNA fragmentation in cultures deprived of glial-conditioned medium. Equal amounts of DNA from sister DIV 3 cultures were $3^{\prime}$ end labeled after 24 or 48 $\mathrm{hr}$ in the indicated condition. Cultures were left undisturbed $(C T R L)$, or were washed into MEMglc $(M E M g)$, or glial-conditioned medium $(G C M)$. End-labeled DNA was separated on a $2 \%$ agarose gel. B, The effect of actinomycin and cycloheximide on neurons deprived of glialconditioned medium. Sister cultures were left undisturbed (CTRL), or washed into MEMglc $(M E M g)$, glial-conditioned medium $(G C M)$, or MEMglc plus actinomycin D $(A C T)$ or cycloheximide $(C H X)$. After 48 $\mathrm{hr}, \mathrm{LDH}$ release by the cultures was measured. Values shown are mean \pm SD for $n=4$. Asterisk (*) indicates statistically significant difference from MEMglc by ANOVA and Student-Newman-Keuls test, $p<0.05$, maximal neuronal LDH released by $24 \mathrm{hr}$ exposure to $500 \mu \mathrm{M}$ NMDA for mixed cultures, or freeze-thaw for pure cultures.

Twenty-four hour exposure to AMPA, kainate, or NMDA. Cultures were washed three times with MEMglc, and receptor agonists (NMDA, 3-300 $\mu \mathrm{M}$; AMPA, 1-100 $\mu \mathrm{M}$; kainate, 3-300 $\mu \mathrm{M}$ ) were added for 24 $\mathrm{hr}$; neuronal death was then quantitated by LDH efflux. The NMDA receptor antagonist, MK-801 $(10 \mu \mathrm{M})$, was included with AMPA or kainate to allow assessment of non-NMDA receptor toxicity without secondary activation of NMDA receptors by release of endogenous glutamate.

Oxygen, glucose-deprivation injury. Cells were placed in an anoxic chamber (Forma, Marietta, OH) in an atmosphere of $85 \% \mathrm{~N}_{2}, 10 \% \mathrm{H}_{2}$, and $5 \% \mathrm{CO}_{2}$, and were deprived of oxygen and glucose by triple exchange of the medium with deoxygenated BSS containing no glucose. Oxygen- and $5.5 \mathrm{~mm}$ glucose-containing BSS was used for control cultures. Cultures were incubated in a $37^{\circ} \mathrm{C}$, humidified incubator in the anoxic chamber for specified periods of time, and then rescued with oxygenated MEMglc. Oxygen in the chamber was $\leq 2 \mathrm{~mm} \mathrm{Hg}$ measured with an oxygen electrode (Microelectrodes, Londonderry, $\mathrm{NH}$ ). At the end of the period of oxygen, glucose-deprivation, cultures were transferred to the $37^{\circ} \mathrm{C}$, normoxic, $5 \% \mathrm{CO}_{2}$ incubator for $24 \mathrm{hr}$, and cell death was determined after $24 \mathrm{hr}$.

${ }^{45} \mathrm{Ca}^{2+}$ accumulation. Cultures were washed twice with HBSS (with $5.5 \mathrm{~mm}$ D-glucose), and then exposed to NMDA $(300 \mu \mathrm{M})$ or kainate in HBSS containing tracer ${ }^{45} \mathrm{Ca}^{2+}(0.5 \mu \mathrm{Ci} /$ culture well; New England Nuclear, Boston, MA) (Hartley et al., 1993). The exposure was terminated after $10 \mathrm{~min}$ by exchanging the medium four times with unlabeled HBSS, followed by lysis of the cells by addition of $0.2 \%$ sodium dodecyl sulfate (SDS). The cells were left in SDS for $2 \mathrm{hr}$, and the lysate was then transferred to scintillation vials. An additional wash of each culture well with SDS was added to the vial, which then underwent $\beta$-counting in duplicate. For kainate exposure, MK-801 was present to prevent secondary activation of NMDA receptors.

Measurement of extracellular glutamate. To determine the concentration of extracellular glutamate, an aliquot of medium was removed from the cultures, and amino acids were derivatized with $o$-phthaldialdehyde/2-mercaptoethanol reagent $(1: 1, \mathrm{vol} / \mathrm{vol})$. Samples were injected onto a Waters Resolve $\mathrm{C} 18$ column, and amino acids separated using a gradient, beginning $4 \mathrm{~min}$ after injection, from $100 \%$ 0.1 M potassium acetate, $\mathrm{pH} 7.35 /$ methanol $20 \%(80: 20)$ to $100 \%$ methanol/0.1 M potassium acetate $\mathrm{pH} 7.35(80: 20)$ over $7 \mathrm{~min}$, a modification of the technique described by Lindroth and Mopper (1979). Amino acids were detected with a fluorescence detector, and commercial standards were injected to identify peaks and allow quantitation based on peak area.

Reagents and solutions. Endotoxin-free water, glutamine, and Minimal Essential Medium (MEM, 330-1430) were obtained from GIBCO (Grand Island, NY). Fetal bovine serum and horse serum came from Hyclone Laboratories (Logan, UT). Laminin was purchased from Collaborative Research, Inc. (Bedford, MA). Epidermal growth factor (EGF), Hoechst dye 33258, propidium iodide, Banderiera simplicifolia I lectin linked to horseradish peroxidase and all other chemicals for tissue culture were purchased from Sigma (St. Louis, MO); trans-PDC was from Tocris Neuramin (Bristol, England). Falcon plasticware came from Becton Dickinson (Lincoln, NJ). MK-801 was from Merck, Sharp and Dohm (Essex, PA). NSE and GFAP antibodies were obtained from Incstar (Stillwater, MN); antibodies to MAP 2 and terminal deoxynucleotidyl transferase were from Boehringer-Mannheim (Indianapolis, IN). Fluorescent secondary antibodies were tagged with BODIPY (Mo-

number sign (\#) indicates significant difference from CTRL. $C$, The percentage of apoptotic cells increased over $48 \mathrm{hr}$ after removing glialconditioned medium. Neuronal cultures were stained with Hoechst dye 33258 and counted 24 and $48 \mathrm{hr}$ after medium change into MEMglc, GCM, or while left undisturbed (CTRL). Cells were scored as apoptotic or diffusely fluorescent, according to nuclear morphology. Numbers shown are apoptotic cells as a percentage of the total cells counted per field, mean \pm SEM for counts of five or six $40 \times$ microscope fields, containing 100-200 cells each, for each condition. Asterisk $(*)$ indicates statistically significant difference, $p<0.05$ by ANOVA and StudentNewman-Keuls test, compared to control for the same time. Number sign (\#) Indicates significant difference from the same condition at 24 hr. 

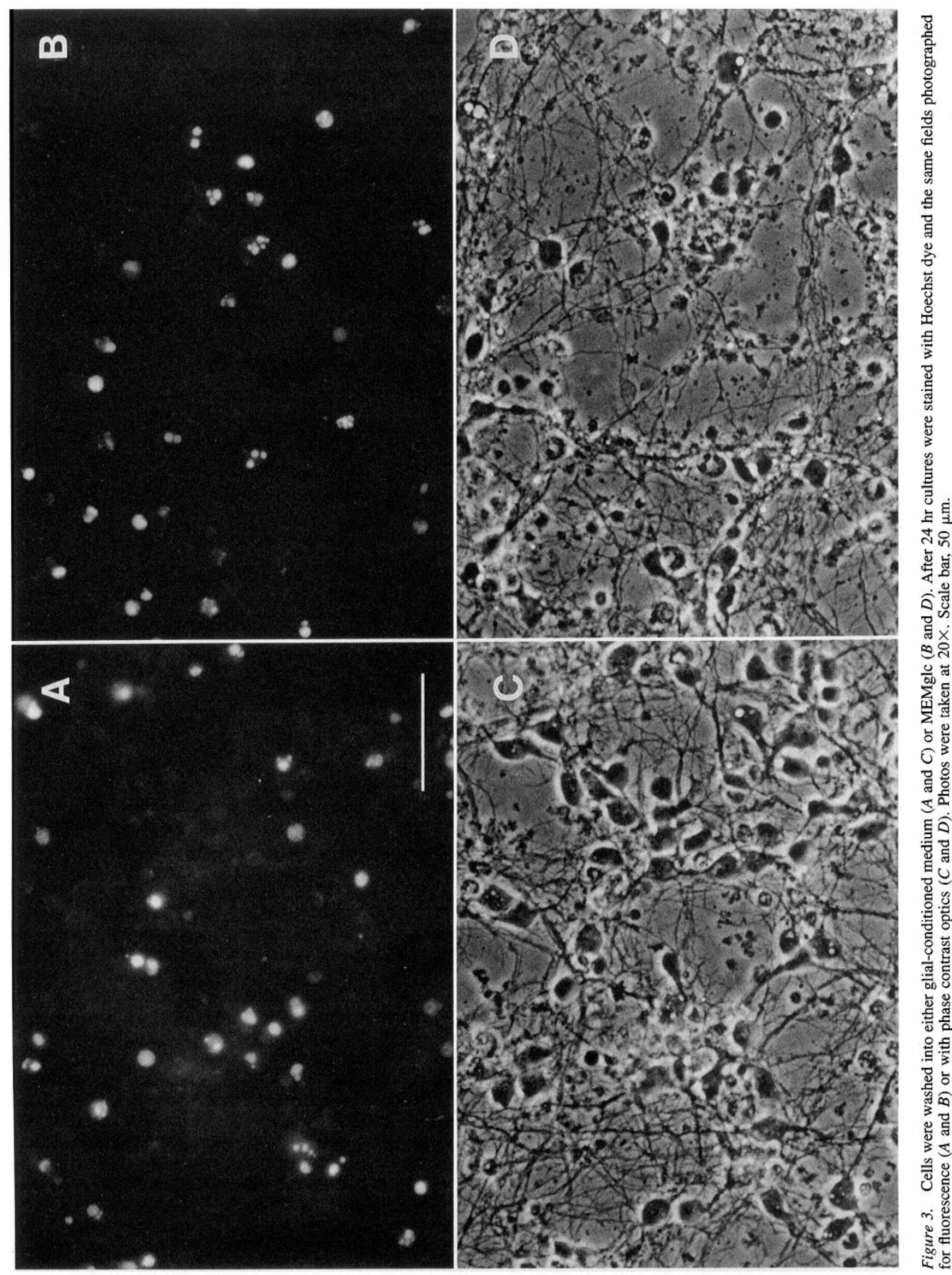
lecular Probes, Eugene, OR) or Cy3 (Jackson ImmunoResearch, West Grove, PA)

Balanced salt solution (BSS) consisted of (in $\mathrm{mM}$ ) $\mathrm{NaCl}, 116 ; \mathrm{KCl}$, $5.4 ; \mathrm{MgSO}_{4}, 0.8 ; \mathrm{NaH}_{2} \mathrm{PO}_{4}, 1.0 ; \mathrm{CaCl}_{2}, 1.8 ; \mathrm{NaHCO}_{3}, 26$; with or without $5.5 \mathrm{mM}$ D-glucose. HEPES-buffered BSS (HBSS) was prepared similarly, except that $10 \mathrm{~mm}$ HEPES (free acid) replaced the $\mathrm{NaHCO}_{3}$, and $\mathrm{NaCl}$ was increased to $132 \mathrm{~mm}$. The medium was brought to $\mathrm{pH} 7.4$ using $\mathrm{NaOH}$. Plating medium (PM) was MEMglc (MEM with $23 \mathrm{~mm}$ glucose) supplemented with glutamine to $2 \mathrm{~mm}, 5 \%$ fetal bovine serum, and $5 \%$ horse serum for neuronal and mixed cultures. Astrocyte cultures were plated in medium containing $10 \%$ fetal bovine serum, $10 \%$ horse serum, and $10 \mathrm{ng} / \mathrm{ml}$ EGF. Glial-conditioned medium (GCM) was prepared by incubating MEMglc with confluent astrocyte cultures grown in $75 \mathrm{~cm}^{2}$ flasks for 2 weeks. After harvest, the medium was filtered for sterility and stored at $4^{\circ} \mathrm{C}$.

\section{Results}

Development of neurons is similar with or without glia

The morphology of neurons grown in pure culture was essentially the same as neurons grown on a bed of astrocytes (Fig. $1 A, C$ ), as demonstrated by MAP 2 or NSE (data not shown) staining at the level of light microscopy. GFAP-immunoreactive cells constituted $<1 \%$ of the cells in the pure neuronal cultures (Fig. 1D, Table 1), in contrast to the confluent monolayer of cells seen in mixed cultures (Fig. $1 B$, Table 1). Cell counts for both types of culture are given in Table I at day in vitro (DIV) 2 and DIV 12. Staining with Banderiera simplicifolia I lectin for microglia revealed only 10-20 microglia per well in mixed cultures, and no microglia in the pure ncuronal cultures examined (data not shown). Mixed and neuronal cultures had similar numbers of NADPH-diaphorase positive cells $(<1 \%$ of neurons), in agreement with earlier results (Koh and Choi, 1988). Both types of cultures had 10-15\% neurons with calcium-conducting AMPA/kainate receptors, as demonstrated by cobalt staining (data not shown), consistent with a previous report by Turetsky et al. (1994)

The development of functional glutamate receptors, as defined by vulnerability to excitotoxic injury and LDH efflux, is essentially complete by DIV 11 in pure neuronal cultures. Cultures exposed to NMDA for 10 min showed little toxicity at DIV 8. By DIV 11, however, the percentage of neurons killed by exposure to NMDA was not statistically different from that seen in DIV 14 cultures (Table 2). In addition, DIV 11 neuronal cultures demonstrated ${ }^{45} \mathrm{Ca}^{2+}$ accumulation during 10 min exposure to NMDA or glutamate (Table 3) that was similar to that reported in a previous study (Hartley et al., 1993). Kainate and AMPA receptor-mediated neurotoxicity in neuronal cultures also was essentially maximal by DIV 11 (not shown). The development of vulnerability to excitotoxic neuronal injury paralleled that seen in sister mixed cultures.

\section{Glial-conditioned medium suppresses apoptosis}

Immature cultures, DIV 2-4, showed an absolute requirement for glial-conditioned medium to survive (Fig. 2B). Since the number of contaminating glial cells in these cultures is quite low, the glial-conditioned medium likely supplies an essential growth or survival factor(s). Astrocytes are known to release factors that promote neuronal survival (Giulian et al., 1993), including brain-derived neurotrophic factor (BDNF; Zafra et al., 1992). Furthermore, several types of neurons and glia (reviews Oppenheim, 1991; Raff et al., 1993; Johnson and Deckwerth, 1993) are known to undergo apoptosis on the removal of a growth or survival factor. We observed that withdrawal of glialconditioned medium from pure cortical neuronal cultures induced apoptosis. In DIV 2 cultures, DNA fragmentation was
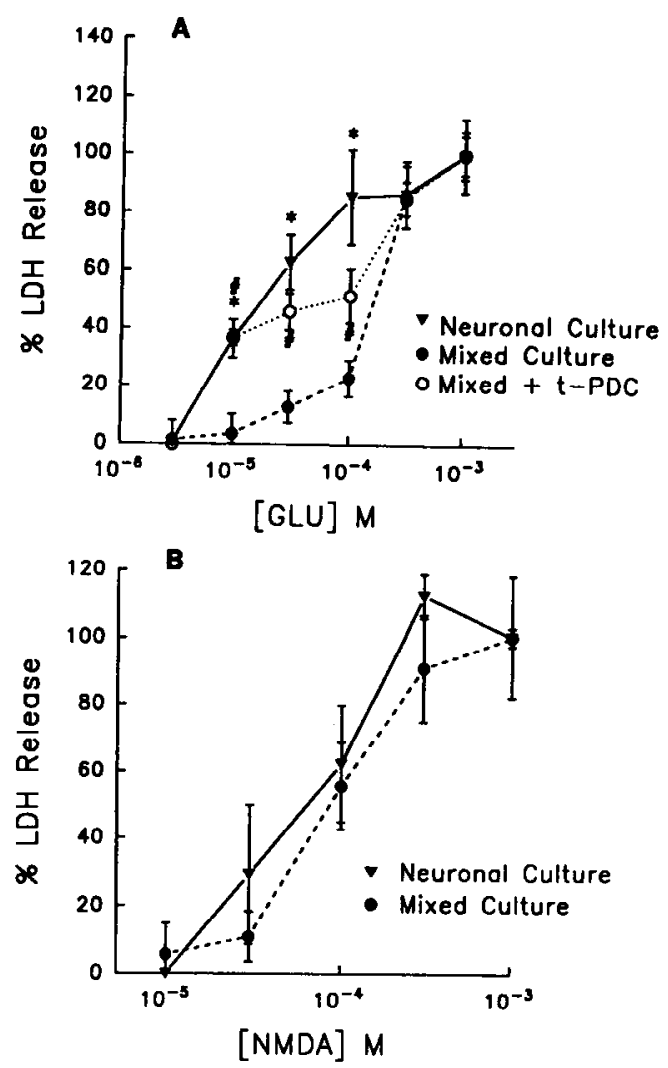

Figure 4. Dose response for $10 \mathrm{~min}$ glutamate or NMDA toxicity. A, Neuronal cultures are approximately 10 times more sensitive to glutamate than mixed cultures. Addition of the glutamate uptake inhibitor, trans-PDC to mixed cultures shifted the dose-response curve back towards that of pure neurons. Values shown are mean \pm SEM for $n=$ 4 for mixed and trans-PDC, $n=16$ for pure neuronal cultures, results representative of three experiments. Significant difference at $p<0.05$ between mixed cultures and pure neuronal cultures at the same concentration of glutamate by ANOVA and Student-Newman-Keuls test is shown by an asterisk $\left({ }^{*}\right)$. Difference between mixed cultures and mixed plus t-PDC is shown by a number sign (\#). B, Dose response for 10 min NMDA toxicity. Pure and mixed cultures show similar responses to brief exposure NMDA toxicity, with no statistically significant difference. Values shown are mean \pm SEM for $n=4$, representative of three similar experiments.

detected by $3^{\prime}$ end labeling 24 and $48 \mathrm{hr}$ after exchange of the glial-conditioned medium with MEMglc (Fig. 2A). Increased numbers of cells with brightly fluorescent, condensed, lobulated nuclei typical of apoptosis (Belizario et al., 1993) were present when cultures were stained with Hoechst dye beginning at 24 $\mathrm{hr}$ of deprivation, and clearly increasing in number at $48 \mathrm{hr}$ (Figs. $2 C, 3 B$ ). Degenerative morphological changes, especially affecting cell processes, are apparent with phase contrast microscopy by $24 \mathrm{hr}$ (Fig. 3D). The survival of cells at $48 \mathrm{hr}$ was substantially increased by inhibition of macromolecular synthesis with either cycloheximide or actinomycin D (Fig. 2B).

\section{Comparative sensitivity to excitotoxicity}

Pure neurons ( $>$ DIV 11) were about 10 -fold more sensitive to brief glutamate exposure than were mixed cultures, which contain approximately $70 \%$ glial cells (Fig. 4A). This differential sensitivity could be reduced by adding the specific glutamate uptake inhibitor, trans-PDC, to mixed cultures to block glial uptake of glutamate. Although trans-PDC may also reduce neuronal uptake of glutamate, as has been shown in hippocampal 

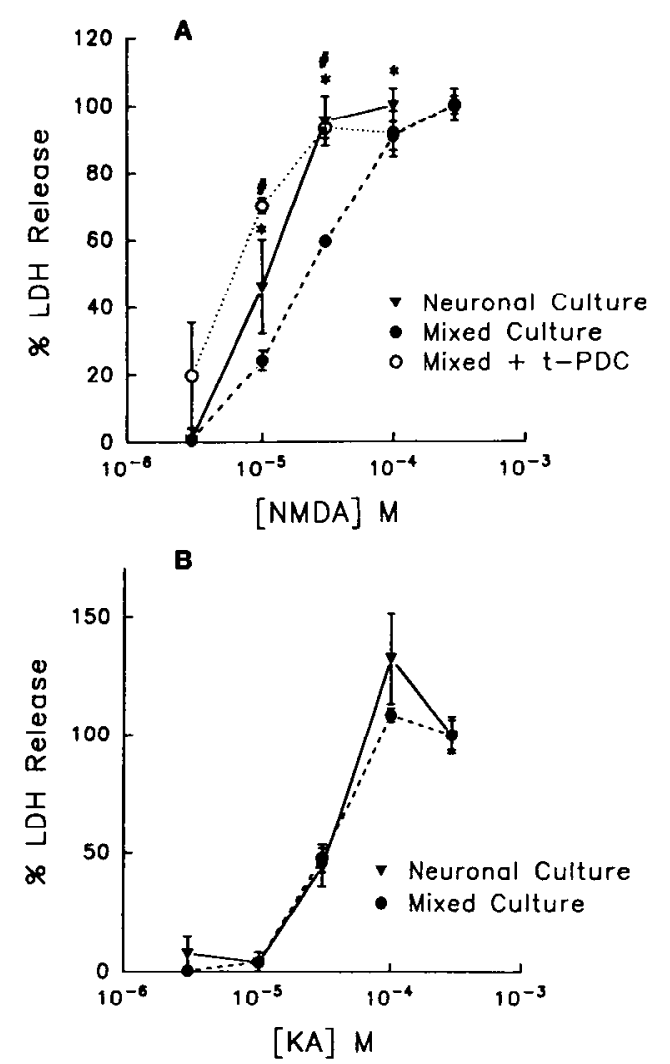

Figure 5. Comparative dose-response curves for $24 \mathrm{hr}$ exposure to NMDA or kainate. $A$. The dose-response curve for NMDA in neuronal cultures is shifted somewhat to the left, possibly reflecting secondary release of glutamate, which is blunted by glial uptake in mixed cultures. Co-incubation with $100 \mu \mathrm{M}$ trans-PDC shifted the dose-response curve of mixed cultures towards that of pure neurons. Values shown are mean \pm SEM for $n=4$, results are representative of three such experiments. Asterisk $(*)$ indicates significant difference between pure and mixed cultures at the same concentration of NMDA by ANOVA and StudentNewman-Keuls test. Number sign (\#) indicates difference between mixed cultures with and without trans-PDC. $B$, The $\mathrm{EC}_{50}$ of kainate in the presence of $10 \mu \mathrm{M}$ MK801 in neuronal cultures is the same as in mixed cultures. Values shown are mean \pm SEM for $n=4$, representative of three such experiments.

slice (Isaacson and Nicoll, 1993), pure neuronal cultures incubated with trans-PDC showed no shift in their dose-response curve (data not shown), suggesting that this is a minor uptake mechanism, or that there is little bath glutamate present in neuronal cultures. The sensitivity of pure neuronal cultures and mixed cultures (Fig. $4 B$ ) to 10 min NMDA toxicity, however, was similar and was not significantly modified by treatment of the cultures with the glutamate uptake inhibitor (not shown).

Cortical neurons require prolonged $(>1 \mathrm{hr}$ ) exposure to nonNMDA receptor agonists to induce neuronal cell death (Koh et al., 1990). Non-NMDA receptor-mediated injury, and the injury produced by prolonged exposure to low concentrations of NMDA, result in insults with characteristics that are somewhat similar to each other, but are different from injury produced by 10 min exposure to high concentrations of NMDA or glutamate (Rose et al., 1991). We therefore determined the sensitivity of pure neurons to $24 \mathrm{hr}$ exposure to NMDA, as well as kainate and AMPA. Pure neurons were more sensitive to injury produced by prolonged exposure to NMDA, and the sensitivity of mixed cultures is significantly increased by trans-PDC (Fig. 5A). The $\mathrm{EC}_{50}$ values for kainate in neuronal and mixed cultures were

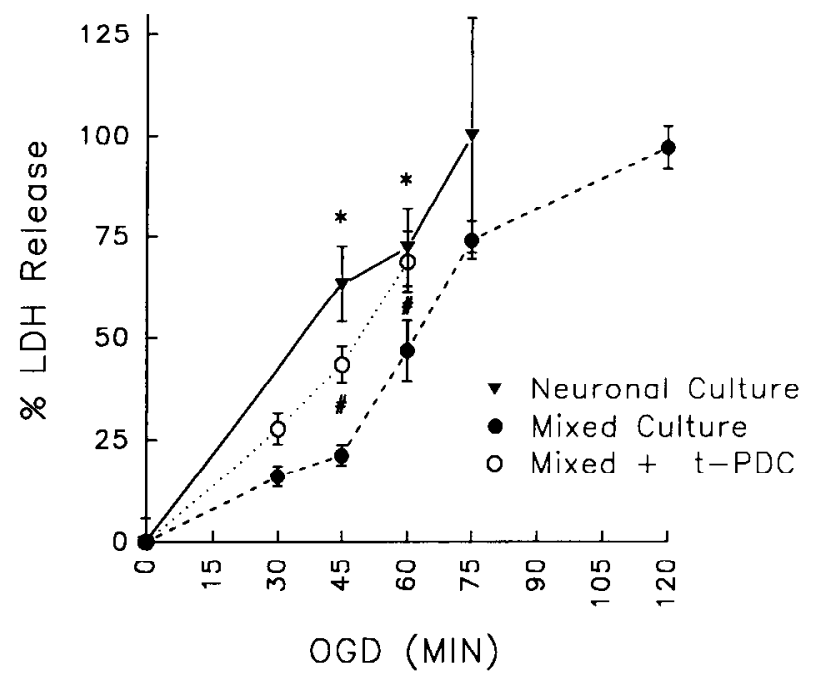

Figure 6. Oxygen, glucose deprivation (OGD) injury of pure neuronal cultures and mixed cultures. Pure neuronal cultures are more sensitive to injury than those containing glia. Addition of trans-PDC increases the sensitivity of mixed cultures. Values shown are mean \pm SEM for $n=4-8$, representative of data from three experiments. Asterisk $\left({ }^{*}\right)$ indicates significant difference between pure and mixed cultures for the same duration of oxygen, glucose deprivation by ANOVA and StudentNewman-Keuls test, $p<0.05$. Number sign $(\#)$ indicates difference between mixed cultures with and without trams-PDC.

indistinguishable (Fig. $5 B$ ). The $\mathrm{EC}_{51}$ concentrations for mixed cultures exposed to NMDA for $10 \mathrm{~min}$ or $24 \mathrm{hr}$, glutamate for $10 \mathrm{~min}$, or kainate for $24 \mathrm{hr}$ were similar to those reported previously in mixed cultures (Koh and Choi, 1988).

In mixed cortical cell cultures, combined oxygen, glucosedeprivation injury has been shown to be mediated, in large part, by NMDA receptors (Kaku et al., 1991; Goldberg and Choi, 1993). In keeping with the greater vulnerability of pure neuronal cultures to NMDA toxicity, a shorter duration of combined oxygen, glucose deprivation was required to produce injury in pure neurons than was necessary for mixed cultures (Fig. 6). When mixed cultures were deprived of oxygen and glucose in the presence of trans-PDC, the curve shifted towards that of neurons alone. However, for neuronal cultures, as for mixed cultures, the oxygen, glucose-deprivation dose response varies more from plating to plating than do the glutamate agonist injury paradigms. In subsequent experiments, we have determined that the density of neuronal cultures is a critical determinant of vulnerability to oxygen, glucose-deprivation injury, and neuronal cultures that are less dense than those used for the reported experiments have been somewhat more resistant to oxygen, glucosedeprivation insult.

\section{AMPA toxicity is exacerbated by glia}

Surprisingly, mixed cultures were more sensitive to AMPA-induced injury than were pure neurons (Fig. 7). To determine whether extracellular glutamate levels might account for this finding, levels of glutamate in the media of cultures exposed to AMPA were measured. Glutamate levels in glial cultures were found to be elevated $2 \mathrm{hr}$ after AMPA (Table 4). This was not due to glial cell death, as exposure to concentrations of AMPA up to $1 \mathrm{~mm}$ for $24 \mathrm{hr}$ or $48 \mathrm{hr}$ did not produce injury, as determined by LDH efflux or light microscopic morphology (data not shown). Others have reported a lack of gliotoxicity after $48 \mathrm{hr}$ exposure to $2 \mathrm{~mm}$ AMPA (Bridges et al., 1992). Media from 


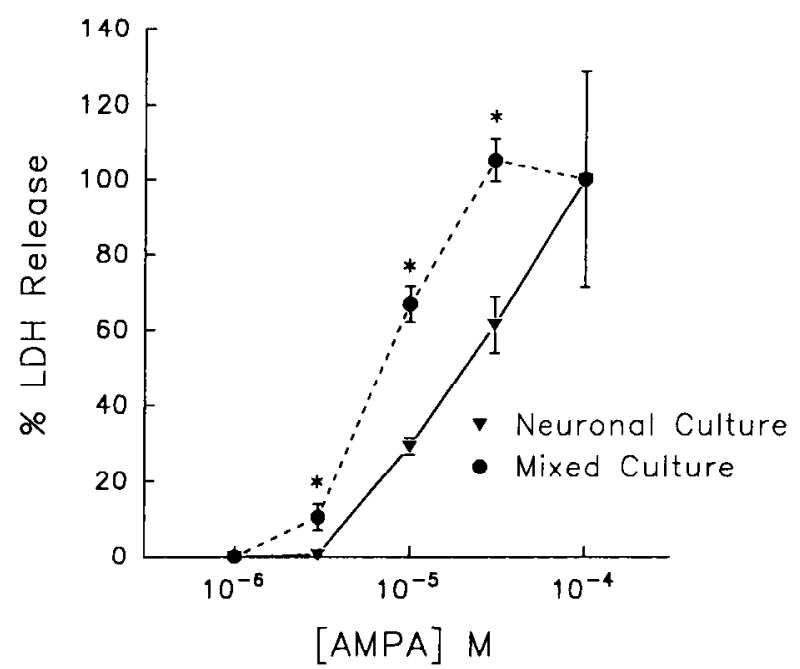

Figure 7. Neurons in the presence of astrocytes are approximately threcfold more sensitive to AMPA toxicity than are neurons alone. Values shown are mean \pm SEM for $n=4$, representative of four experiments. Asterisk (*) indicates statistically significant difference between pure and mixed cultures at the same concentration of AMPA with $p<$ 0.05 by ANOVA and Student-Newman-Keuls test. By taking the ratio of the $\mathrm{EC}_{50}$ (estimated graphically) for the four pairs of neuronal and mixed cultures, mixed cultures were found to be $3.08 \pm 0.99$ times more sensitive to AMPA than pure neuronal cultures (mean $\pm \mathrm{SD} ; p$ $<0.05$ by $t$-test; $95 \%$ CI were 1.5 and 4.65 ).

pure neuronal cultures exposed to AMPA also showed a small increase in glutamate (Table 4). However, glutamate levels in mixed cultures exposed to AMPA were not elevated after $2 \mathrm{hr}$. Exposure of glial cultures to NMDA or kainate failed to increase glutamate in the medium. No glutamate determinations were done for AMPA exposures longer than $2 \mathrm{hr}$, when neuronal death begins to occur, since the amount of glutamate present would be increasingly confounded by glutamate from dead cells, and would no longer reflect glutamate released by astrocytes and neurons in response to AMPA.

\section{Discussion}

In establishing the conditions for the growth of murine cortical neurons without supporting glial cells, we observed that removal of glial-conditioned medium from immature cultures led to their death. This was likely due to apoptosis, as it was accompanied by internucleosomal cleavage of DNA, showed condensed nuclear morphology when stained with Hoechst 33258 dye, and was inhibited by macromolecular synthesis inhibitors. The time course of this induction was consistent with the findings of others studying sympathetic neurons acutely deprived of NGF (Martin et al., 1988). Cerebral astrocytes can be stimulated to produce BDNF by glutamate in combination with a $\beta$-adrenergic agonist ( Zafra et al., 1992), and BDNF has been shown to improve survival of cortical neurons under certain conditions (Ghosh et al., 1994). Basic tibroblast growth factor (bFGF), another trophic factor that acts on central neurons (Morrison et al., 1986), can be induced in cultured rat astrocytes by exposure to glutamate (Pechan et al., 1993). What factors are present in glialconditioned medium is not yet known.

Murine neuronal cultures lacking glia were more sensitive to glutamate toxicity than neurons in the presence of glia, in agreement with previous work in rat cultures (Rosenberg and Aizenman, 1989; Rosenberg et al., 1992; Robinson et al., 1993). Ad-
Table 4. Concentration of glutamate in the media of cultures exposed to glutamate receptor agonists

\begin{tabular}{|c|c|c|c|}
\hline Culture type & Condition & $\begin{array}{l}{[\mathrm{Glu}](\mu \mathrm{M}) \text { in }} \\
\text { medium (mean } \pm \\
\text { SEM) }\end{array}$ & $n$ \\
\hline \multirow[t]{8}{*}{ Glial } & Control & $0.39 \pm 0.16^{*}$ & 12 \\
\hline & AMPA & $1.36 \pm 0.64$ & 8 \\
\hline & $10 \mu \mathrm{M}$ & $2 . .38 \pm 1.06$ & 7 \\
\hline & $30 \mu \mathrm{M}$ & $4.53 \pm 1.69 *$ & 8 \\
\hline & $100 \mu \mathrm{M}$ & $4.19 \pm 1.34^{*}$ & 12 \\
\hline & Kainate $300 \mu \mathrm{M}$ & $0.37 \pm 0.09$ & 7 \\
\hline & NMDA $100 \mu \mathrm{M}$ & $0.12 \pm 0.06$ & 4 \\
\hline & $300 \mu \mathrm{M}$ & $0.07 \pm 0.05$ & 4 \\
\hline \multirow[t]{2}{*}{ Neuronal } & Control & $0.38 \pm 0.09$ & 7 \\
\hline & AMPA $30 \mu \mathrm{M}$ & $0.72 \pm 0.13 \dagger$ & 7 \\
\hline \multirow[t]{3}{*}{ Mixed } & Control & $0.23 \pm 0.11$ & 8 \\
\hline & AMPA $30 \mu \mathrm{M}$ & $0.50 \pm 0.26$ & 7 \\
\hline & $100 \mu \mathrm{M}$ & $0.45 \pm 0.13$ & 8 \\
\hline
\end{tabular}

Glutamate was measured in the medium of cultures exposed to the indicated conditions for $2 \mathrm{hr}$. This time point precedes the onset of cell death, as measured by release of LDH into the medium, or loss of trypan blue exclusion. We routinely included MK-80I $(10 \mu \mathrm{M})$ with either AMPA or kainate exposure in mixed cultures to eliminate secondary activation of NMDA receptors by endogenously released glutamate. Therefore, MK-801 was included with AMPA or kainate for all experiments.

* Different from glial control, $p<0.05$ by ANOVA and Student-NewmanKeuls test.

$\dagger$ Different from pure neuronal control, $p<0.05$ by ANOVA and StudentNewman-Keuls test.

dition of a glutamate uptake inhibitor (trans-PDC) to mixed cultures increased their vulnerability to glutamate, consistent with the hypothesis that the differential sensitivity of pure neuronal and mixed cultures is due, in part, to glutamate uptake by glia. Pure neurons were also somewhat more sensitive to oxygen, glucose deprivation injury, in agreement with a prior report (Vibulsreth et al., 1987), and inhibition of glial glutamate uptake by trans-PDC increased the sensitivity of mixed, but not pure neuronal cultures, to oxygen, glucose deprivation injury.

Toxicity from 10 min exposure to NMDA was not significantly altered by the presence of astrocytes. NMDA, unlike glutamate, is not taken up by glia to an appreciable extent (Drejer et al., 1982; Brew and Attwell, 1987). In contrast, NMDA exposure for $24 \mathrm{hr}$ was somewhat greater in pure neuronal cultures compared to mixed cultures. This excess vulnerability was reproduced in mixed cultures by the addition of trans-PDC, suggesting that endogenous release of glutamate may contribute to this slower form of NMDA injury. Injury produced by brief exposure to high concentrations of NMDA may differ from that produced by chronic exposure to lower concentrations of NMDA (Rose et al., 1991). The importance of endogenous glutamate release to injury may be one way in which these two insults diverge. Toxicity from prolonged ( $24 \mathrm{hr}$ ) exposure to kainate was not affected by the presence of astrocytes. This observation can be explained by lack of substantial accumulation of bath glutamate in our cultures after addition of kainate (Dugan, unpublished observations).

In contrast to the neuroprotective role of astrocytes in many excitotoxic injuries, the presence of astrocytes actually enhanced AMPA-induced neurotoxicity, suggesting that the interaction between neurons and glia in excitotoxicity is complex. That there is cross talk between neurons and glial is now well established. 
Rapid inward currents in astrocytes have been observed with electrical activity in neighboring neurons (Murphy et al., 1993). Using Fura-2 photomicrography, multiple glial calcium spikes were observed with continuous stimulation of retinal nerve axons (Kriegler and Chiu, 1993); these calcium spikes are similar to those reported in astrocytes in response to glutamate (CornellBell et al., 1990). A recent report of the effects of glia on neurotransmission in hippocampal culture demonstrated neuronal currents after direct glial depolarization (Mennerick and Zorumski. 1994), possibly reflecting glial glutamate release. Increased neuronal calcium in response to astrocyte stimulation was reported by both Nedergaard (1994) and Parpura et al. (1994). The latter study directly demonstrated glutamate release by glia exposed to bradykinin, and suggested that the concentration of released glutanate from glia was sufficient to stimulate neuronal glutamate receptors, specifically NMDA receptors, as the neuronal calcium increase was blocked by the NMDA receptor antagonist, AP-5. AMPA activation of astrocytes might result in similar glutamate release.

We postulated that reversal of astrocyte glutamate transport might explain the unexpected observation that pure neuronal cultures were less susceptible to AMPA toxicity than cultures containing a high proportion of astrocytes. The neuroprotective function of the glutamate transporter to remove excess extracellular glutamate might fail under conditions of energy failure or astrocyte depolarization (Nicholls and Attwell, 1990; Huang et al., 1993), resulting in loss of the transmembrane sodium gradient, and reversal of the glutamate transporter (Kaupinnen et al., 1988). Glutamate might also be released from astrocytes by an exocytotic process analogous to that demonstrated for acetylcholine release from muscle (Dan and Poo, 1992), or by astrocyte swelling (Kimelberg et al., 1990). Thus, extracellular glutamate in mixed cultures exposed to AMPA might derive not only from neurons, but from astrocytes, compounding the neurotoxic action of AMPA on the neurons. We found that AMPA induced release of glutamate from astrocyte cultures, consistent with a previous report of glutamate release from type-2 astrocytes in response to kainate or quisqualate (Levi and Patrizio, 1992). However, glutamate levels in mixed cultures exposed to AMPA were as low as levels in pure neuronal cultures, arguing against astrocytic glutamate release as an explanation for the enhanced vulnerability of mixed cultures to AMPA.

AMPA-induced glutamate release by astrocytes in pure glial cultures, but not in mixed cultures, might be explained by neuronal modulation of glial glutamate receptors. Cultured cortical astrocytes possess AMPA/kainate receptors (Sontheimer et al., 1988; Glaum et al., 1990; Jensen and Chiu, 1990; Condorelli et al., 1993; Holzwarth et al., 1994), and the complement of these receptors on astrocytes can vary by brain region and can be affected by culture conditions and stage of development (Hatten, 1985; Barres et al., 1990; Pearse, 1993). There is precedent for neurons determining expression of glial ion channels. The expression of calcium channels on astrocytes, for example, is affected by the presence of neurons in the culture (reviewed by Sontheimer, 1994). Further, it is possible that an astrocytic response to AMPA other than glutamate release might feed back on neurons and be deleterious.

An alternative explanation for enhanced AMPA vulnerability of mixed cultures is at the level of neuronal AMPA receptor number or composition. Modulation of the number, or subunit composition, of neuronal AMPA receptors by astrocytes might produce a more toxic complement of neuronal receptors. To our knowledge, there are, to date, no specific reports of astrocytic modification of glutamate receptor expression. However; this intriguing possibility provides a viable explanation of our results, and is a hypothesis that we are currently exploring.

The results reported here suggest that glia modify AMPAmediated injury in mixed cultures by unexpectedly worsening injury. Whatever the mechanism whereby this occurs, these findings provide a novel additional mechanism for the protection seen with non-NMDA agonists in animal models of ischemia (Sheardown et al., 1990; Buchan et al., 1991), in that they may have important actions on glial glutamate receptors in addition to their effects on neuronal receptors.

\section{References}

Aizenman Y, deVellis J (1987) Brain neurons develop in a serum and glial free environment: effects of transferrin, insulin, insulin-like growth factor-I and thyroid hormone on neuronal survival, growth and differentiation. Brain Res 406:32-42.

Barres BA, Koroshetz WJ, Chun LLY, Corey DP (1990) Ion channel expression by white matter glia: the type 1 astrocyte. Neuron 5:527544.

Belizario JE, Tilly JL, Sherwood SW (1993) Caffeine potentiates the lethality of tunour necrosis factor in cancer cells. Br J Cancer 67 : 1229-1235.

Borg J, Spitz B, Hamel G, Mark J (1985) Selective culture of neurons from rat cerebral cortex: morphological characterization, glutamate uptake and related enzymes during maturation in various culture media. Dev Brain Res 18:37-49.

Brew H, Attwell D (1987) Electrogenic glutamate uptake is a major current carrier in the membrane of axolotl retinal glial cells. Nature 327:707-709.

Bridges RJ, Stanley MS, Anderson MW, Cotman CW, Chamberlin AR (1991) Conformationally defined neurotransmitter analogues. Selective inhibition of glutamate uptake by one pyrrolidine 2,4 dicarbox ylate diastereomer. J Med Chem 34:717-725.

Bridges RJ, Hatalski CG, Shim SN, Cummings BJ, Vijayan V, Kundi A, Cotman CW (1992) Gliotoxic actions of excitatory annino acids. Neuropharmacology 31:899-907.

Buchan AM, Li H, Cho S, Pulsinelli WA (1991) Blockade of the AMPA receptor prevents CAI hippocampal injury following severe but transient forebrain ischemia in adult rats. Neurosci Lett 132:255258.

Choi DW, Rothman SM (1990) The role of glutamate neurotoxicity in hypoxic-ischemic neuronal death. Annu Rev Neurosci 13:171-182.

Choi DW, Maulucci-Gedde MA, Kreigstein AR (1987) Glutamate neurotoxicity in cortical cell culture. J Neurosci 7:357-368.

Colton CA, Abel C, Patchett J, Keri J, Yao J (1992) Lectin staining of cultured CNS microglia. J Histochem Cytochem 40:505 512.

Condorelli DF, Dell'Albani P, Corsaro M, Barresi V. Giuffrida Stella AM (1993) AMPA-selective glutamate receptor subunits in astroglial cultures. J Neurosci Res 36:344-356.

Cornell-Bell AH, Finkbeiner SM, Cooper MS, Smith SJ (1990) Glutamate induces calcium waves in cultured cortical astrocytes: longrange glial signalling. Science 247:470-473.

Dan Y, Poo M (1992) Quantal transmitter secretion from myocytes loaded with acetylcholine. Nature 359:733-736.

Dichter MA (1978) Rat cortical neurons in cell culture: culture methods, cell morphology, electrophysiology, and synapse formation. Brain Res 149:279-293.

Drejer J, Larsson OM, Shousboe A (1982) Characterization of L-glutamate uptake into and release from astrocytes and neurons cultured from different brain regions. Exp Brain Res 47:259 269.

Fox PT, Raichle ME, Mintun MA, Dence C (1988) Non-oxidative glucose consumption during focal physiologic neural activity. Science 241:462-464.

Ghosh A, Carnahan J, Greenberg ME (1994) Requirement for BDNF in activity-dependent survival of cortical neurons. Science 263:16181623.

Giffard RG, et al. (1992) Anesthesiology 77:A740.

Giulian D, Vaca K, Corpuz M (1993) Brain glia release factors with opposing actions upon neuronal survival. J Neurosci 13:29-37.

Glaum SR, Holzwarth JA, Miller RJ (1990) Glutamate receptors ac- 
tivate $\mathrm{Ca}^{2+}$ mobilization and $\mathrm{Ca}^{2+}$ influx into astrocytes. Proc Natl Acad Sci USA 87:3454-3458.

Goldberg MP, Choi DW (1993) Combined oxygen and glucose deprivation in cortical cell culture: calcium-dependent and calciumindependent mechanisms of neuronal injury. J Neurosci 13:3510 3524.

Hartley DM, Kurth MC, Bjerkness L, Weiss JH, Choi DW (1993) Glutamate receptor-induced ${ }^{4.5} \mathrm{Ca}^{2+}$ accumulation in cortical cell culture correlates with subsequent neuronal degeneration. J Neurosci 13: 1993-2000.

Hatten ME (1985) Neuronal regulation of astroglial morphology and proliferation in vitro. J Cell Biol 100:384-396.

Hewett SJ, Czernansky CA, Choi DW (1994) Potentiation of NMDAinduced neuronal injury following induction of astrocytic iNOS in mixed cortical cell cultures. Neuron 13:487-494.

Holzwarth JA, Gibbons SJ, Brorson JR, Philipson LH, Miller RJ (1994) Glutamate receptor agonists stimulate diverse calcium responses in different types of cultured rat cortical glial cells. J Neurosci 14:18791891

Huang R. Shuaib A, Hertz L (1993) Glutamate uptake and glutamate content in primary cultures of mouse astrocytes during anoxia, substrate deprivation and simulated ischemia under normothermic and hypothermic conditions. Brain Res 618:346-351

Isaacson JS. Nicoll RA (1993) The uptake inhibitor I.trans-PDC enhances responses to glutamate but fails to alter the kinetics of excitatory synaptic currents in the hippocampus. J Neurophysiol 70:2187 2191

Jensen AM, Chiu SY (1990) Fluorescence measurement of changes in intracellular calcium induced by excitatory amino acids in cultured cortical astrocytes. J Neurosci 10:1165-1175.

Johnson EM Jr, Deckwerth TL (1993) Molecular mechanisms of developmental neuronal death. Annu Rev Neurosci 16:31-46.

Kaku DA, Goldberg MP, Choi DW (1991) Antagonism of non-NMDA receptors augments the neuroprotective effect of NMDA receptor blockade in cortical cultures subjected to prolonged deprivation of oxygen and glucose. Brain Res 554:344-347.

Kauppinnen RA, McMahon HT. Nicholls DG (1988) $\mathrm{Ca}^{2+}$-dependent and $\mathrm{Ca}^{2+}$-independent glutamate release, energy status and cytosolic free $\mathrm{Ca}^{2}$ concentration in isolated nerve terminals following metabolic inhibition: possible relevance to hypoglycaemia and anoxia. Neuroscience 27:175-182.

Kimelberg HK, Goderie SK, Higman S, Pang S, Waniewski RA (1990) Swelling-induced release of glutamate, aspartate, and taurine from astrocyte cultures. J Neurosci 10:1583-1591.

Koh JY, Choi DW (1987) Quantitative determination of glutamate mediated cortical neuronal injury in cell culture by lactate dehydrogenase efflux assay. J Neurosci Methods 20:83-90.

Koh JY, Choi DW (1988) Vulnerability of cultured cortical neurons to damage by excitotoxins: differential susceptibility of neurons containing NADPH-diaphorase. J Neurosci 8:2 I53-2163.

Koh JY, Goldbcrg MP, Hartlcy DM, Choi DW (1990) Non-NMDA receptor-mediated neurotoxicity in cortical culture. J Neurosci 10 : 693-705.

Kriegler S, Chiu SY (1993) Calcium signalling of glial cells along mammalian axons. J Neurosci 13:4229-4245.

Levi G, Patrizio M (1992) Astrocyte heterogeneity: endogenous amino acid levels and release evoked by non- $N$-methyl-D-aspartate receptor agonists and by potassium-induced swelling in type- 1 and type-2 astrocytes. J Neurochem 58:1943-1952.

Lindroth P, Mopper K (1979) High performance liquid chromatograph ic determination of subpicomolar amounts of amino acids by precolumn fluorescence derivatization with $o$-phthaldialdehyde. Anal Chem $51: 1667-1674$.

Martin DP, Schmidt RE, DiStefano PS, Lowry OH, Carter JG, Johnson EM Jr (1988) Inhibitors of protein synthesis and RNA synthesis prevent neuronal death caused by nerve growth factor deprivation. $J$ Cell Biol 106:829-844.

Meldrum B, Garthwaite J (1990) Excitatory amino acid neurotoxicity and neurodegenerative disease. Trends Pharmacol Sci 11:379-387

Mennerick S, Zorumski CF (1994) Glial contributions to excitatory neurotransmission in cultured hippocampal cells. Nature 368:59-62.

Morrison RS. Sharma A, DeVellis J, Bradshaw RA (1986) Basic fibroblast growth factor supports the survival of cerebral cortical neurons in primary culture. Proc Natl Acad Sci USA 83:7537-7541.

Murphy TH, Blatter LA, Wier WG, Baraban JM (1993) Rapid com- munication between neurons and astrocytes in primary cortical cultures. J Neurosci 13:2672-2679.

Nedergaard M (1994) Direct signaling from astrocytes to neurons in cultures of mammalian brain cells. Science 263:1768-1771.

Nicholls D, Attwell D (1990) The release and uptake of excitatory amino acids. Trends Neurosci 11:462-468.

Oppenheim RW (1991) Cell death during development of the nervous system. Annu Rev Neurosci 14:45.3-501.

Parpura V, Basarsky TA, I iu F, Jeftinija K, Jeftinija $S$, Haydon $P C$ (1994) Glutamate-mediated astrocyte-neuron signalling. Nature 369: $744-747$.

Pearce B (1993) Amino acid receptors. In: Astrocytes-pharmacology and function (Murphy S, ed), pp 47-66. San Diego: Academic.

Pechan PA, Chowdhury K, Gerdes W, Seifert W (1993) Glutamate induces the factors NGF, bFGF, the receptor FGF-RI and $c$-fos mRNA expression in rat astrocyte culture. Neurosci Lett 15.3:111114.

Pruss RM, Akeson RL, Racke MM, Wilburn JL (1991) Agonist-activated cobalt uptake identifies divalent cation-permeable kainate receptors on neurons and glial cells. Neuron 7:509-518.

Quach TT, Rose C, Schwartz JC (1978) ['H]Glycogen hydrolysis in brain slices: responses to neurotransmitters and modulation of noradrenaline receptors. J Neurochem 30:1335-1341.

Raff MC (1992) Social controls on cell survival and cell death. Nature 356:397-400.

Robinson MB, Djali S, Buchhalter JR (1993) Inhibition of glutamate uptake with 1-trans-pyrrolidine-2,4-dicarboxylate potentiates glutamate toxicity in primary hippocampal cultures. J Neurochem 61 : 2099-2103.

Rose K, Bruno VMG, Oliker R, Choi DW (1990) Nordihydroguaiaretic acid (NDGA) attenuates slow excitatory amino acid-induced neuronal degeneration in cortical cultures. Soc Neurosci Abstr 16:288

Rose K, Goldberg MP, Choi DW (1992) Cytotoxicity in murine neocortical cell culture. In: Methods in toxicology, Vol 1, In vitro bio logical systems (Tyson CA, Frazier JM, eds). pp 46-60. New York: Academic

Rosenberg PA (1991) Accumulation of extracellular glutamate and neuronal death in astrocyte-poor cortical cultures exposed to glutamine. Glia 4:91-100.

Rosenberg PA, Aizenman E (1989) Hundred-fold increase in neuronal vulnerability to glutamate toxicity in astrocyte-poor cultures of rat cerebral cortex. Neurosci Lett 103:162-168.

Rosenberg PA, Amin S, Leitner M (1992) Glutamate uptake disguises neurotoxic potency of glutamate agonists in cerebral cortex in dissociated cell culture. J Neurosci 12:56-61.

Sanfeliu C, Hunt A, Patel AJ (1990) Exposure to $N$-methyl-D-aspartate increases release of arachidonic acid in primary cultures of rat hippocampal neurons and not in astrocytes. Brain Res 526:241-248.

Scherer-Singler U, Vincent SR, Kimura H, McGeer EG (1983) Demonstration of a unique population of neurons with NADPH-diaphorase histochemistry. J Neurosci Methods 9:29-34.

Sheardown MJ, Nielsen EO, Hansen AJ, Jacobsen P, Honore T ( 1990 ) 2,3-Dihydroxy-6-nitro-7-sulfamoy]-benzo(F)quinoxaline: a neuroprotectant for cerebral ischemia. Science 247:571-574.

Sontheimer H, Kettenmann H, Backus KHM (1988) Glutamate opens $\mathrm{N} \cdot \mathrm{d} / \mathrm{K}$ channels in cultured astrucyles. Glia $1: 328-3,36$.

Sugiyama K, Brunori A, Mayer ML (1989) Glial uplake of excitatory amino acids influences neuronal survival in cultures of mouse hippocampus. Neurosci 32:779-791.

Swanson RA, Yu ACH, Chan PH, Sharp FR (1990) Glutamate increases glycogen content and reduces glucose utilization in primary astrocyte cultures. J Neurochem 54:490-496.

Tilly JL, Hsueh AJW (1993) Microscale autoradiographic method for the qualitative and quantitative analysis of apoptotic DN $\wedge$ fragmen tation. J Cell Physiol 154:519-526.

Turetsky DM, Canzoniero LMT, Sensi SL, Weiss JH, Goldberg MP, Choi DW (1994) Cortical neurons exhibiting kainate-activated $\mathrm{Co}^{2-}$ uptake are selectively vulnerable to AMPA/kainate receptor-mediated toxicity. Neurobiol Dis, in press.

Vibulsreth S, Hefti F, Ginsberg MD, Dietrich WD, Busto R (1987) Astrocytes protect cultured neurons from degeneration induced by anoxia. Brain Res 422:303-311.

Volterra A, Trotti D, Cassutti P, Tromba C, Salvaggio A, Melcangi RC. Racagni G (1992) High sensitivity of glutamate uptake to extracel- 
Jular free arachidonic acid levels in rat cortical synaptosomes and astrocytes. J Neurochem 59:600-606.

Wallen CA, Higashikubo R, Roti Roti JL (1983) Comparison of the cell kill measured by the Hoechst-propidium iodide flow cytometric assay and the colony formation assay. Cell Tissue Kinet 16:357-365.

Yu ACH, Hertz E, Hertz L (1984) Alterations in uptake and release rates for GABA, glutamate, and glutamine during biochemical mat- uration of highly purified cultures of cerebral cortical neurons, a GABAergic preparation. J Neurochem 42:951-960.

Zafra F, Lindholm D, Castren E, Hartikka J, Thoenen H (1992) Regulation of brain-derived neurotrophic factor and nerve growth factor mRNA in primary cultures of hippocampal neurons and astrocytes. J Neurosci 12:4793-4799. 